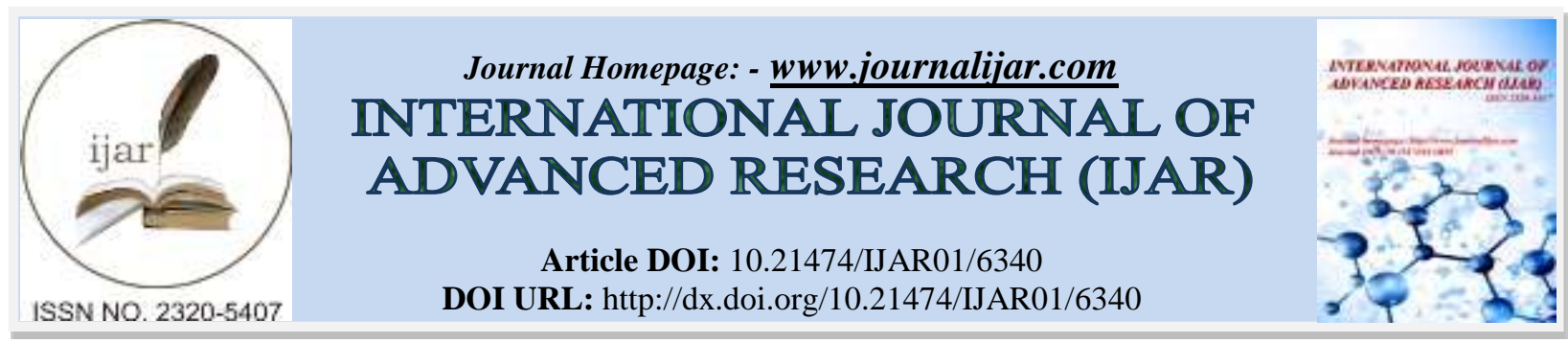

RESEARCH ARTICLE

\title{
CANDIDA ALBICANS: CAN IT BE A PERIODONTAL PATHOGEN?
}

Dr. Babitha G.A ${ }^{1}$, Dr.Vangara Aditya ${ }^{2}$, Dr. Shobha Prakash ${ }^{3}$, Dr. Suresh.K ${ }^{4}$ and Dr. Kishore G.Bhat ${ }^{5}$.

1. Professor in Department of Periodontics, College of Dental Sciences, Davangere, Karnataka - 577004, India.

2. Post Graduate Student in Department of Periodontics, College of Dental Sciences, Davangere, Karnataka - 577004, India.

3. Professor and Head of Department, Department of Periodontics, College of Dental Sciences, Davangere, Karnataka - 577004, India.

4. Professor, Department of Microbiology, Jagadguru Jayadeva Murugarajendra Medical College, Davangere, Karnataka - 577004, India.

5. Professor, Department of Microbiology, Maratha Mandal's N.G.H Institute of Dental Sciences \& Research Centre, Belgaum, Karnataka - 590019, India.

\section{Manuscript Info}

Manuscript History

Received: 18 November 2017

Final Accepted: 20 December 2017

Published: January 2018

Key words:Real-Time Polymerase Chain Reaction, Aggressive Periodontitis, Chronic Periodontitis.
Candida albicans, Periodontal Pocket,

\section{Abstract}

Background: The term periodontitis encompass several polymicrobial infectious diseases of multifactorial etiology, with chronic and aggressive forms. In spite of the etiopathogenic differences between these two forms of the disease, few studies have analyzed the subgingival colonization by yeast. It is currently unclear if yeasts participate in the etiology of this disease and if they show specificity for either the chronic or aggressive forms.

Aim and Objectives: To explore and quantify the presence of Candida albicans in Chronic Periodontitis (CP), Aggressive Periodontitis (AP) and Periodontally Healthy Patients (PH).

Materials and Method: A total of 30 patients were included in this study, Group A:10 patients with Chronic Periodontitis, Group B:10 patients with Aggressive Periodontitis, Group C:10 patients with Periodontal Healthy Patients. The samples were collected from gingival sulcus using Universal Curette before Scaling and Root Planing (SRP) from three groups and sent to lab for investigation to identify Candida albicans by using rt-PCR.

Results: The number of candida albicans were more in $\mathrm{CP}$ followed by AP compared to PH Patients.

Conclusion: It was concluded that candida albicans were associated with $\mathrm{CP}$ and $\mathrm{AP}$ as compared to $\mathrm{PH}$ patients favouring further progression of periodontal disease with high occurrence of deep pockets.

Copy Right, IJAR, 2018,. All rights reserved.

\section{Introduction:-}

The periodontal disease represent a group of localized microbial-induced infections involving the gingival and supporting tissues of the teeth. A periodontal pocket is not a mere pathological deepening of the gingival sulcus but its formation represents the pathological sequel of microbial- and inflammatory-mediated degradation of 
collagenous connective tissues and alveolar bone. ${ }^{1}$ In spite of the polymicrobial character of periodontal disease, the role of yeasts in this illness has received little or no attention. ${ }^{1}$ The Candida spp. are opportunistic pathogens that can cause disease in hosts who are compromised by underlying local or systemic pathological processes. ${ }^{2}$ Chronic periodontitis $(\mathrm{CP})$ has both localized and generalized forms. It is initiated and sustained by a polybacterial infection caused by Porphyromonas gingivalis, Aggregatibacter actinomycetemcomitans and Tannerella forsythia among other species. In addition, host defense mechanisms are also important in the pathogenesis and tissue destruction. $\mathrm{CP}$, the most frequent form of periodontitis, begins at any age although it is more common in adults, and is characterized by its extension (number of sites involved) and severity (amount of clinical insertion loss). Aggressive periodontitis (AP) also presents localized and generalized forms which are characterized by the rapid loss of bone and insertion and the presence of familial aggregation. Localized AP which begins close to puberty, is characterized by the presence of abundant seric antibodies against infectious agents (A.actinomycetemcomitans), and has a specific distribution in teeth of the dental arches. ${ }^{3}$

Candida albicans is the species most often associated with oral lesions, but other Candida spp., including $C$. glabrata, C.tropicalis, C. parapsilosis, C. krusei, and C. dubliniensis have also been isolated in the saliva of subjects with and without oral candidiasis. The isolation of Candida from the oral cavity does not imply the presence of disease. Fungal organisms commonly colonize the tongue, palate, and buccal mucosa. Such colonization may also occur in subgingival plaque of adults with periodontitis. The Candida have virulence factors that facilitate colonization and proliferation in the oral mucosa and, possibly, in periodontal pockets. These fungal organisms can coaggregate with bacteria in dental biofilm and adhere to epithelial cells. These interactions, which are associated with their capacity to invade gingival conjunctive tissue, may be important in microbial colonization that contributes to progression of oral diseases. In addition to these properties, Candida spp also produce enzymes, such as the collagenases and proteinases that degrade extracellular matrix proteins, and immunoglobulins. ${ }^{2}$ However, they may be isolated from the subgingival microbiota in about $17 \%$ of patients with periodontitis. Furthermore, it has been reported that the proportion of yeasts in the periodontal pockets is similar to some bacterial periodontopathogens, suggesting a possible role for Candida spp. in the pathogenesis of the disease..$^{5}$

In spite of the involvement of different etiological agents in chronic and aggressive periodontal disease, few studies analyze the possible role of yeasts in any of these forms of periodontitis. Therefore, the majority of studies that report the presence of yeasts in periodontal pockets do not specify if patients suffer the chronic or aggressive form of the disease. In majority of studies report that the presence of yeasts in periodontal pocket do not specify if patient suffer with chronic or aggressive form of the disease. ${ }^{3}$ In the present study, we had explored to quantify the presence of Candida albicans in chronic Periodontitis (CP), Aggressive Periodontitis (AP) and Periodontally healthy patients $(\mathrm{PH})$ were analyzed, with the purpose of describing possible differences between these groups of individuals with respect to their periodontal condition.

\section{Materials and Method:-}

The patients were taken from Out Patient Department, Department of Periodontics, College of Dental Sciences, Davangere, Karnataka. Patients were explained about the procedure and an informed consent was taken from them.

A total of 30 patients (age group: 25-50 years) were included in this study and were divided into three groups: Group A: 10 Patients with Chronic Periodontitis (CP), Group B: 10 Patients with Aggressive Periodontitis (AP), Group C - 10 Patients with Periodontal healthy patients (PH).Subjects included in this study are Chronic Periodontitis with $>20$ years of age and had at least 14 natural teeth, Probing Depth (PD) $>6 \mathrm{~mm}$ and Loss of Attachment (LOA) $>5 \mathrm{~mm}$, Aggressive Periodontitis with $<30$ years old, had >15 natural teeth Pocket Depth (PD) and loss of attachment (LOA) measurements $>4 \mathrm{~mm}$ localized in the first molars and/or incisors, and three other sites. Patients who were excluded are with history of systemic diseases, Pregnancy, Anti-inflammatory drugs in the 3 months prior to the study, Smoking and Alcohol.

\section{Sample Collection:-}

The samples were collected from gingival sulcus using Universal Curette (Hu-Friedy 4R-4L) [Figure - 1] before SRP from three different groups. The samples were collected in Eppendrof tubes containing TE buffer (Tris-EDTA) medium [Figure - 2, 3] for each patient separately and sent to Maratha Mandal Dental College and Research Centre, Belagavi lab for investigation, to identify Candida albicans by using rt-PCR. A real-time polymerase chain reaction (Real-Time PCR), also known as quantitative polymerase chain reaction (qPCR), is a laboratory technique of molecular biology based on the polymerase chain reaction (PCR). 


\section{Processing Of Samples:-}

A biocabinet containing instruments (Micropipettes, PCR tubes strip) [Figure - 4] were placed and each sample was transferred from eppendrof tubes to PCR tubes strips with help of micropipettes. [Figure - 5]

DNA Extraction Procedure (Modified Proteinase-K method) ${ }^{6}$ :-

The samples were transferred to the tube containing T.E.buffer. Vortex the samples thoroughly. Sonicate the samples at $70 \mathrm{~Hz}$ for 3 minutes. Take 500 microlitre of sonicated sample and Centrifuge at 10,000 rpm for $5 \mathrm{~min}$. Discard the supernatant. Add 500 microliter fresh T.E. buffer centrifuge for 3-4 minutes. [Figure - 6,7,8]

\section{TE Buffer:-}

1M Tris Buffer : $0.5 \mathrm{ml}, 0.5 \mathrm{M}$ EDTA : $100 \mu \mathrm{l}$, Distilled water : made to $50 \mathrm{ml}$. [Figure - 9]

\section{Lysis Buffer I:-}

1M Tris buffer : $500 \mu$ l, Triton X-100 : $500 \mu \mathrm{l}, 0.5 \mathrm{M}$ EDTA: $100 \mu \mathrm{l}$, Distilled Water : Made to $50 \mathrm{ml}$. [Figure - 9]

Lysis II Buffer:-

Tris HCL : $50 \mathrm{mM}\left(\mathrm{p}^{\mathrm{H}} 8.0\right), \mathrm{KCL}: 50 \mathrm{mM}, \mathrm{MgCl}_{2}: 2.5 \mathrm{mM}$, Tween $20: 0.45 \%$, Nodient P-40:0.45\%. [Figure - 9]

Repeat above procedure for 3-4 times with fresh T.E. buffer. Discard supernatant add 50 micro liter lysis buffer I, Vortex it and keep for $5 \mathrm{~min}$. Add 50 microliter Lysis buffer II and 10 micro liter proteinase $-\mathrm{K}$ (100ug/ml), vortex vigorously. Keep it in water bath for $2 \mathrm{hrs}$ then keep in boiling water bath for 10 minutes. Stored the DNA at $-20^{0}$. The PCR conditions were as follows Initial denaturation was done at $95^{\circ} \mathrm{C}, 5$ min denaturation was done at $94^{0} \mathrm{C}, 1$ min, annealing was done at $56^{\circ} \mathrm{C}, 2.30 \mathrm{~min}$ followed by extension at $72^{0} \mathrm{C}, 2 \mathrm{~min}$ around 30 cycles. [Figure - 10, Graph - 1]

\section{Statistical Analysis:-}

Data obtained was entered in Microsoft excel 2010 and statistical analysis was done using SPSS version 21.The statistical test used was One way ANOVA followed by Tukey Post hoc test. The test is statistically significant where $\mathrm{p}$ is $<0.05$.[Graph - 2]

\section{Results:-}

To analyze if the degree of yeast colonization present in the various sites sampled showed differences among the three groups, the CFUs (colony forming units) per subject of yeasts recovered from gingival sulcus and samples were determined. In general terms, the results were variable and showed a heterogeneous distribution with a broad range. ${ }^{3}$ The presence of number of candida albicans was different in each group, and it was highest in patients with chronic periodontitis as compared to aggressive and periodontally healthy patients. It is important to highlight that, Chronic Periodontitis (CP) [Table - 1] who showed the highest CFU value than Aggressive Periodontitis (AP) [Table - 2] and Periodontally Healthy groups (PH) [Table - 3]. 


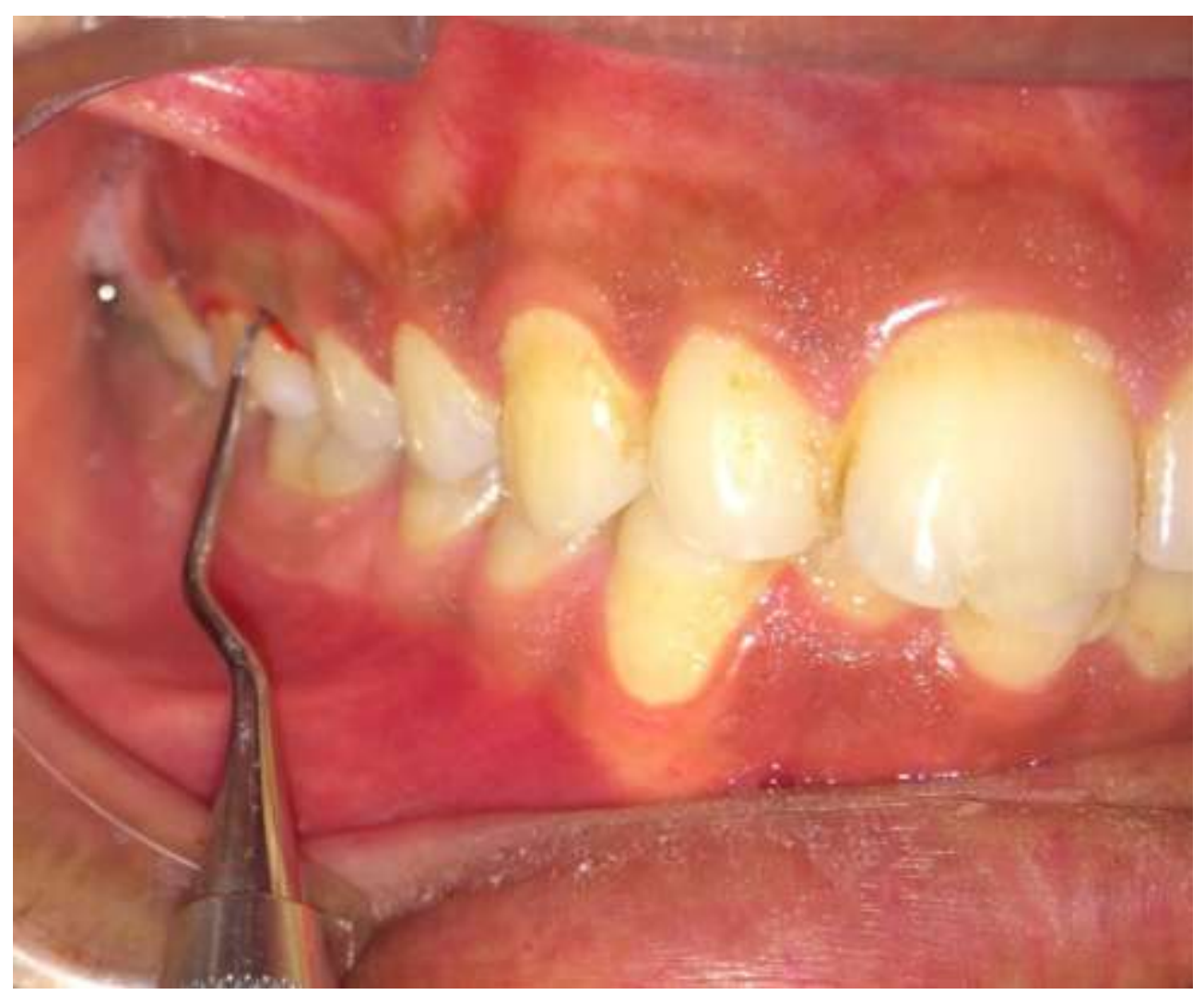

Figure 1:- Universal Curette

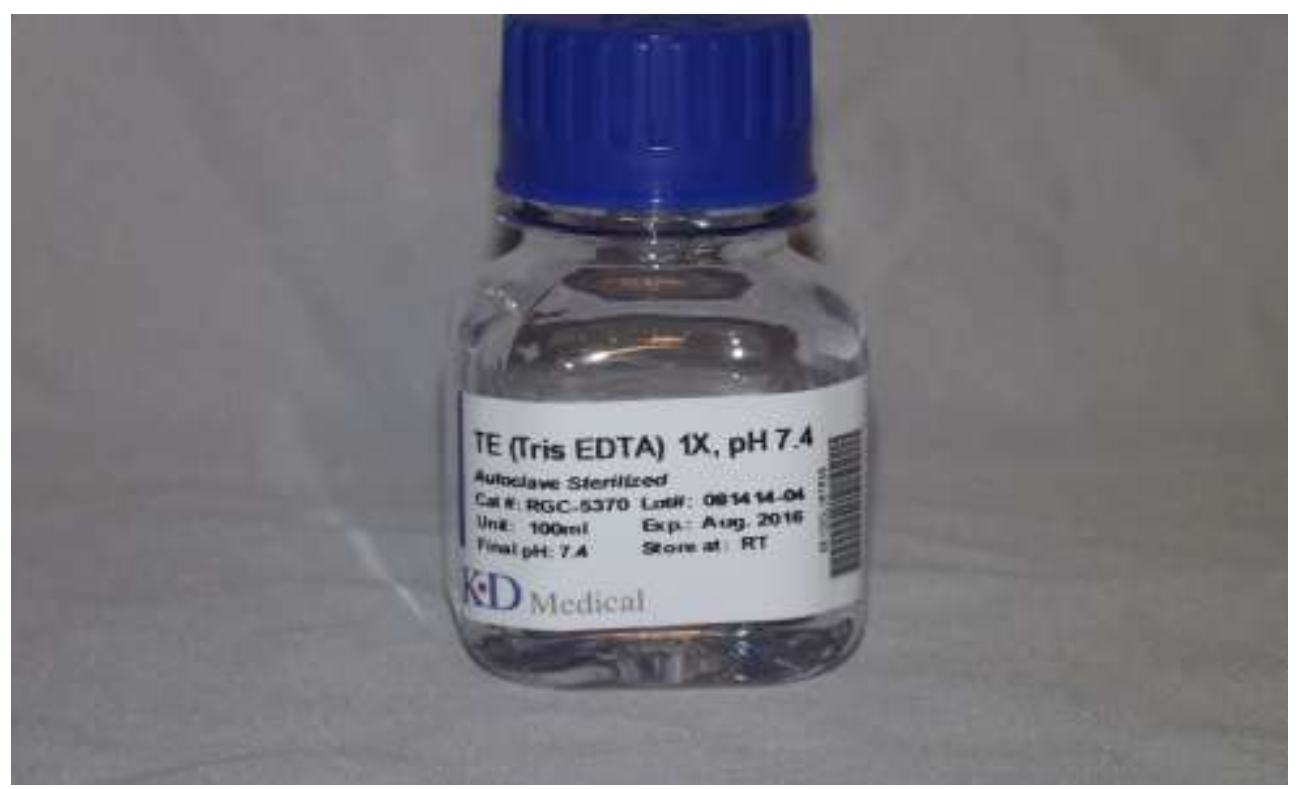

Figure 2:- TE (Tris EDTA) Medium 


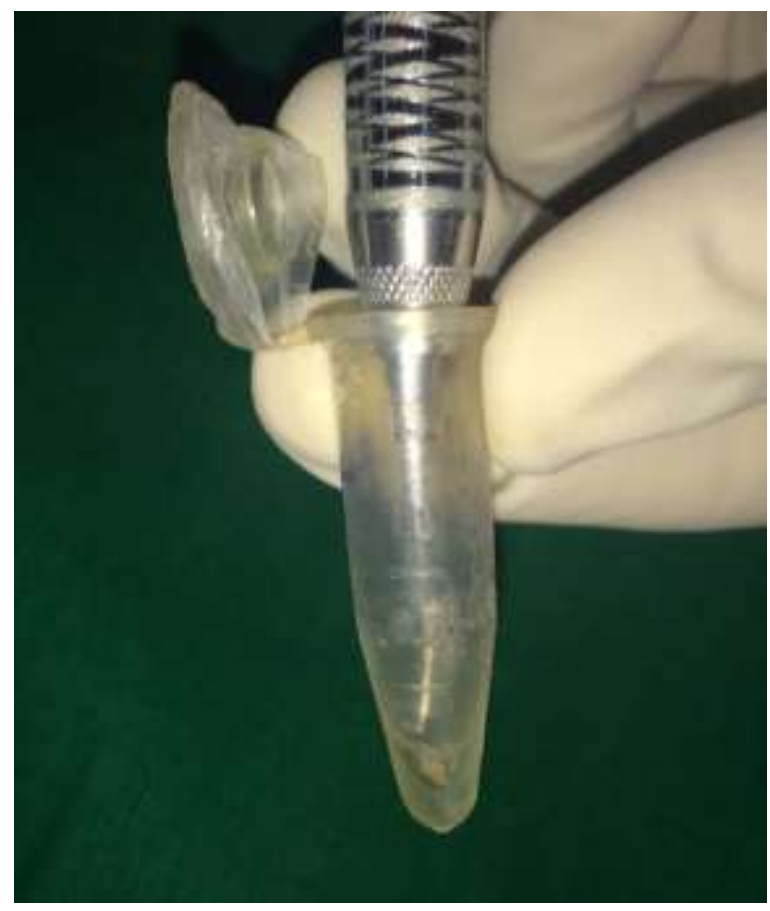

Figure 3:- TE Buffer (Tris-EDTA) Medium along with Sample

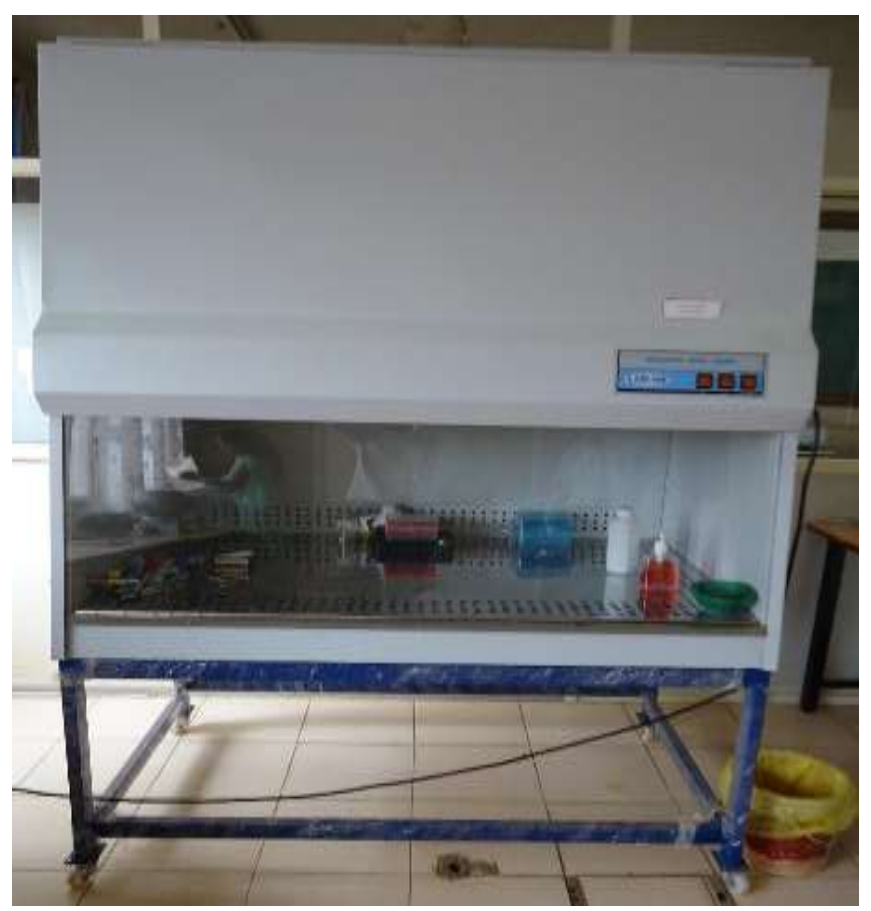

Figure 4:- Biocabinet ventilated laboratory workspace for working with materials (potentially contaminated with) pathogens requiring a defined biosafety level. 


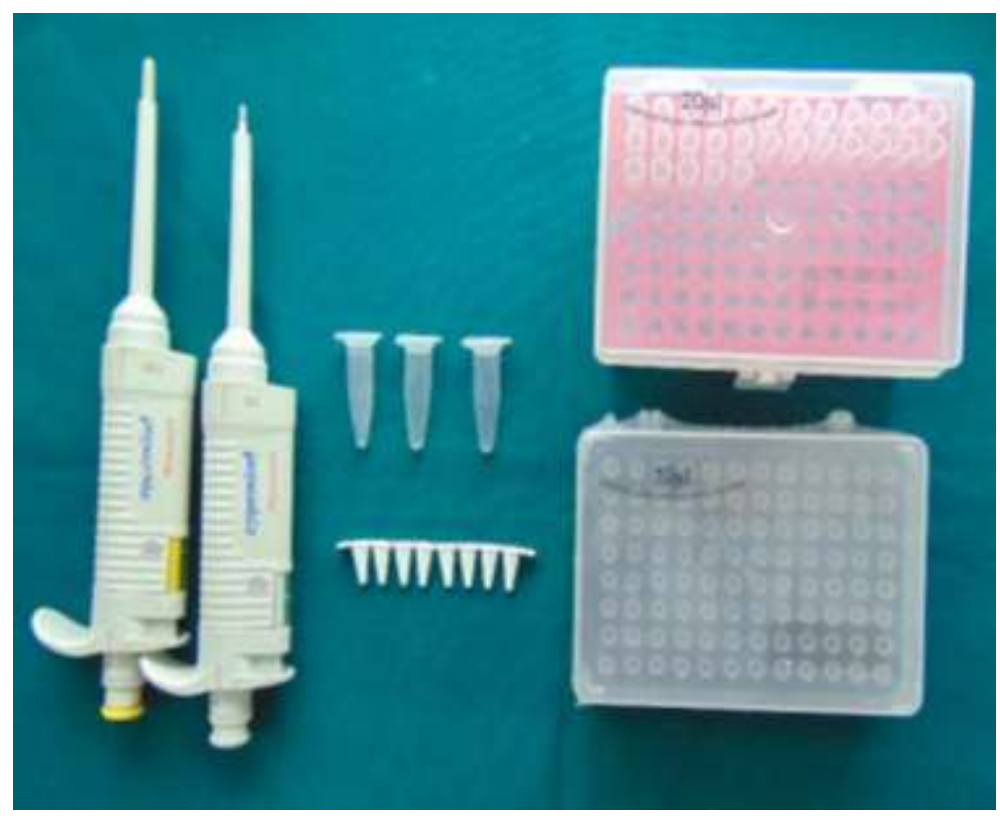

Figure 5:- Micropipettes, PCR tubes strip Eppendrof tubes

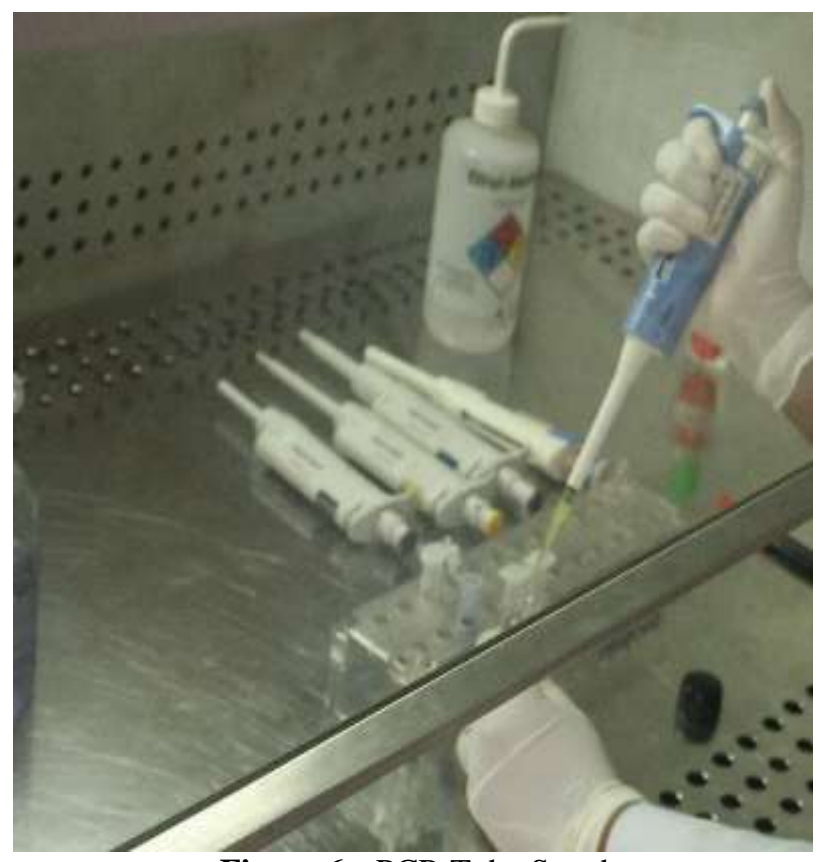

Figure 6:- PCR Tube Stand 


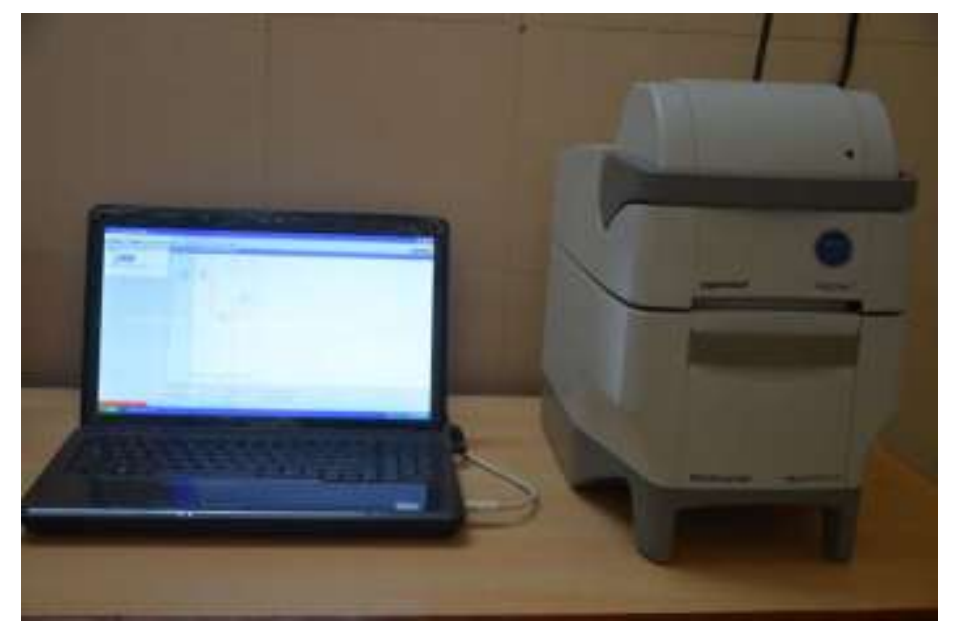

Figure 7:- PCR Machine (Thermal Cycles) \& Data transferred to laptop

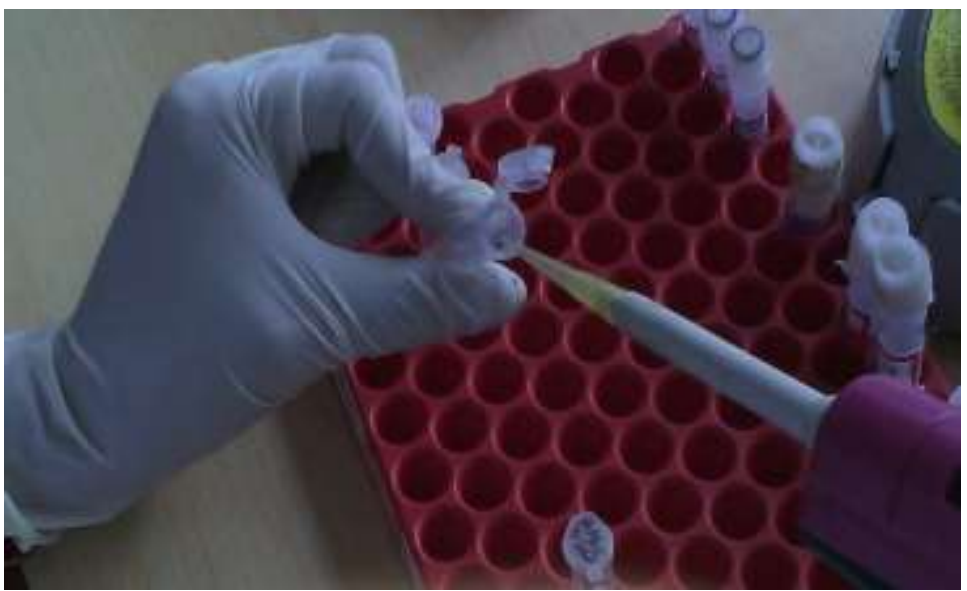

Figure 8:- DNA Extraction Procedure

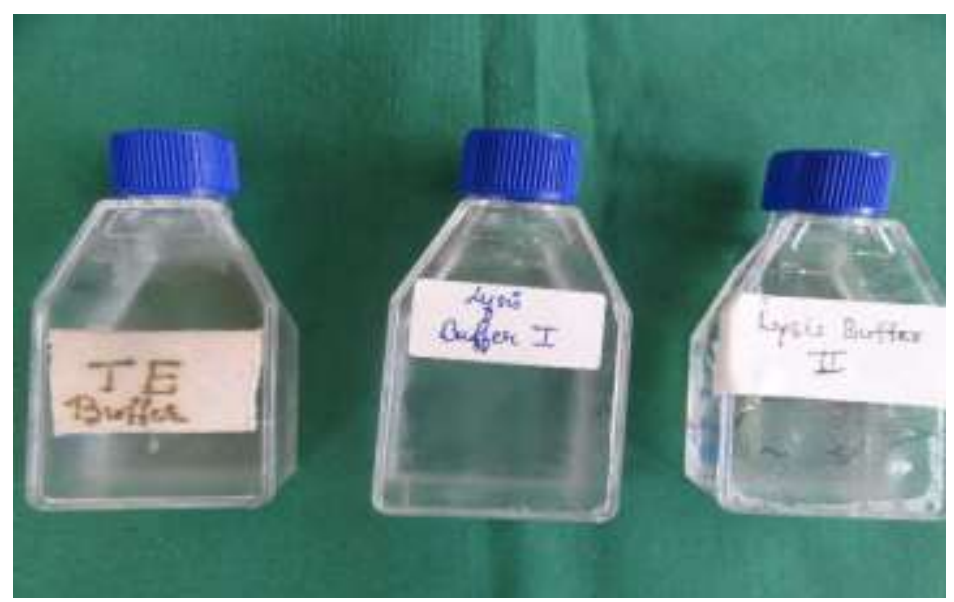

Figure 9:- DNA Extraction Reagents 


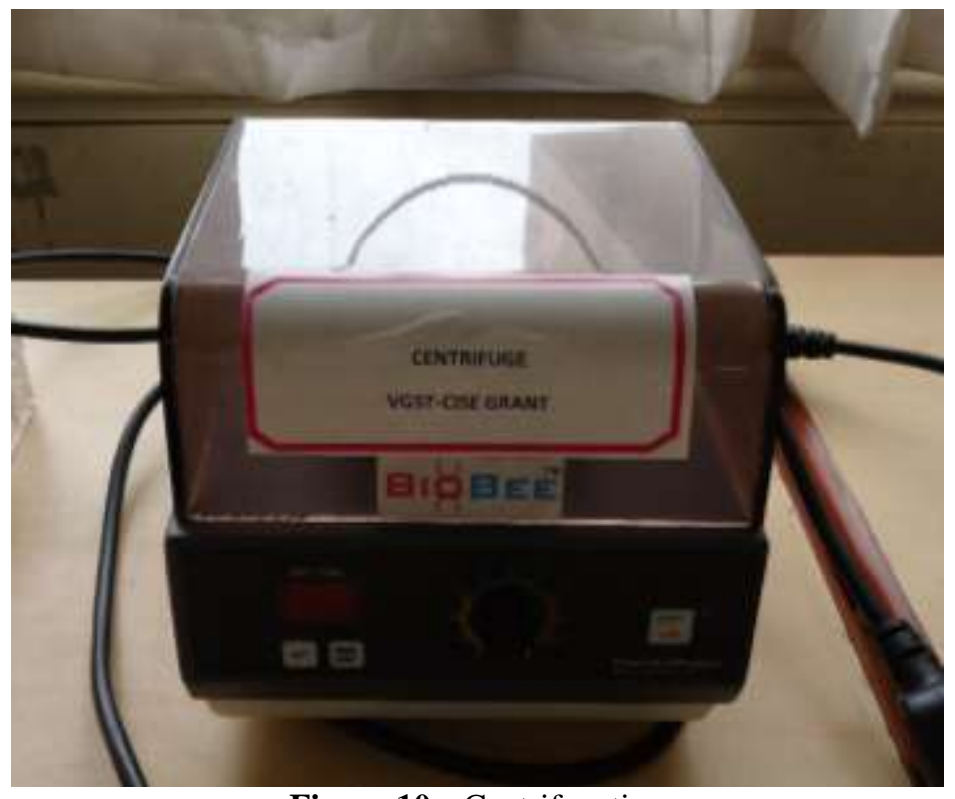

Figure 10:- Centrifugation

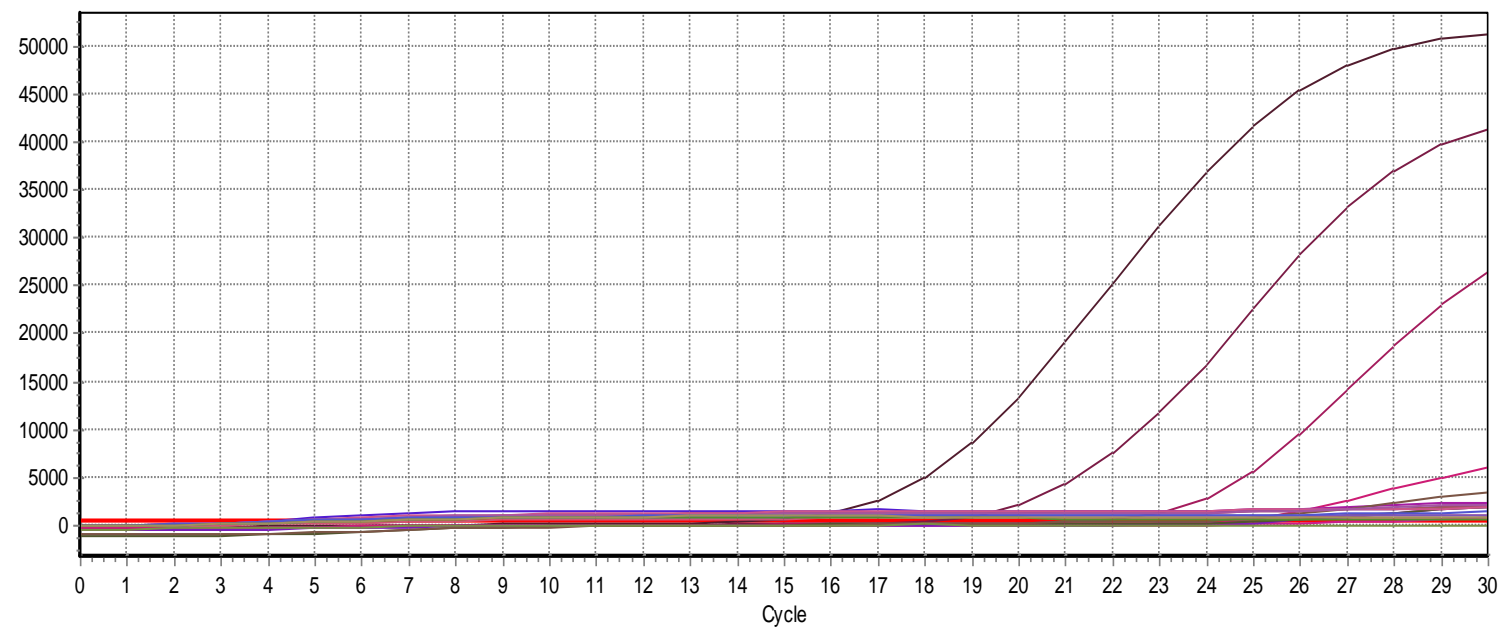

Threshold: $\quad 414$ (Noiseband)

Baseline settings: automatic, Drift correction OFF

Graph 1:- Sample Analysis as graph 


\section{Comparison between the three Groups}

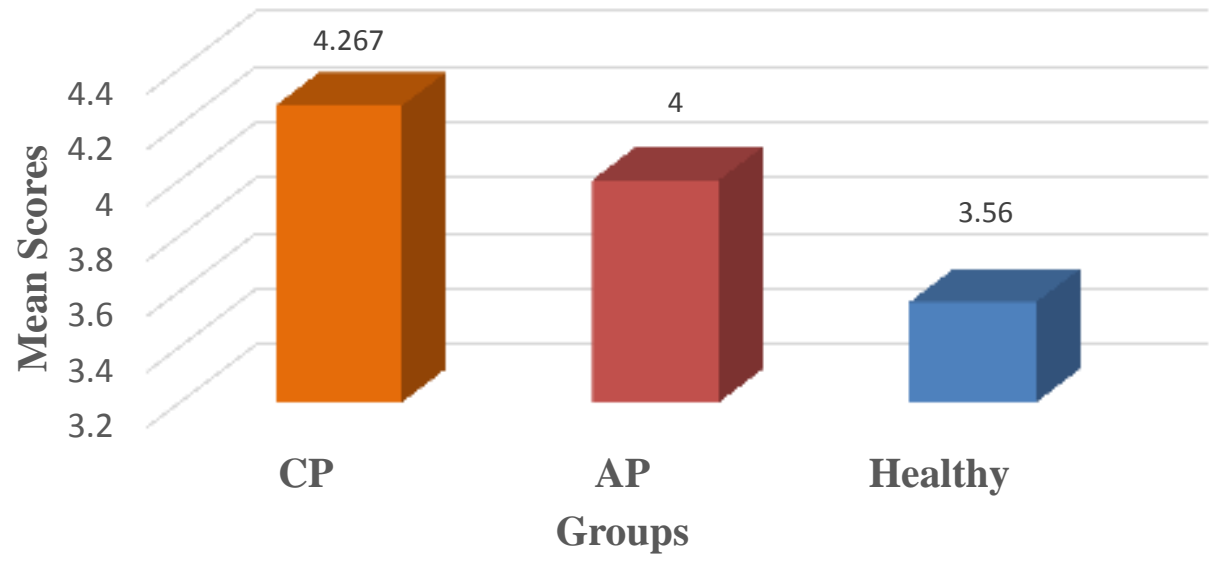

Graph 2:- Statistical Analysis

Table 1:- Group A : Chronic Periodontitis (CP)

\begin{tabular}{|c|c|}
\hline Sl.No & C.albicans $(\mathrm{CFU} / \mathrm{ml})$ \\
\hline 1 & $8.18 \times 10^{4}$ \\
\hline 2 & $1.78 \times 10^{8}$ \\
\hline 3 & $1.42 \times 10^{5}$ \\
\hline 4 & $1.68 \times 10^{7}$ \\
\hline 5 & $6.92 \times 10^{3}$ \\
\hline 6 & $3.84 \times 10^{5}$ \\
\hline 7 & $6.63 \times 10^{7}$ \\
\hline 8 & $4.90 \times 10^{5}$ \\
\hline 9 & $1.80 \times 10^{8}$ \\
\hline 10 & $6.63 \times 10^{7}$ \\
\hline
\end{tabular}

Table 2:- Group B : Aggressive Periodontitis (AP)

\begin{tabular}{|c|c|}
\hline Sl.No & C.albicans (CFU/ml) \\
\hline 1 & $1.64 \times 10^{7}$ \\
\hline 2 & $7.84 \times 10^{6}$ \\
\hline 3 & $7.21 \times 10^{7}$ \\
\hline 4 & $1.21 \times 10^{7}$ \\
\hline 5 & $1.61 \times 10^{8}$ \\
\hline 6 & $1.25 \times 10^{8}$ \\
\hline 7 & $6.35 \times 10^{7}$ \\
\hline 8 & $1.28 \times 10^{8}$ \\
\hline 9 & $5.10 \times 10^{7}$ \\
\hline 10 & $2.08 \times 10^{5}$ \\
\hline
\end{tabular}

Table 3:- Group C: Periodontally Healthy $(\mathrm{PH})$

\begin{tabular}{|c|c|}
\hline Sl. No & C.albicans $(\mathrm{CFU} / \mathrm{ml})$ \\
\hline 1 & $5.49 \times 10^{4}$ \\
\hline 2 & $3.67 \times 10^{5}$ \\
\hline 3 & $4.56 \times 10^{7}$ \\
\hline 4 & $1.04 \times 10^{5}$ \\
\hline
\end{tabular}




\begin{tabular}{|l|l|}
\hline 5 & $1.70 \times 10^{3}$ \\
\hline 6 & $2.82 \times 10^{6}$ \\
\hline 7 & $8.29 \times 10^{7}$ \\
\hline 8 & $7.56 \times 10^{6}$ \\
\hline 9 & $3.41 \times 10^{5}$ \\
\hline 10 & $4.13 \times 10^{7}$ \\
\hline
\end{tabular}

\section{Discussion:-}

Oral cavity plays host to wide array of microorganisms inclusive of variety of bacteria, viruses and fungi. In most individuals; C. albicans resides as part of harmless natural microflora and appears as a transient flora in immunocompromised individuals. It is the causative in majority of cases of mucosal disease and hence the most widely studied species of Candida, under varying circumstances. ${ }^{5}$ In this study, the samples were collected from gingival sulcus of PH subjects and patients with AP and CP were analyzed. In the three groups of subjects studied, the distribution of yeast CFUs was very heterogeneous, indicating that some subjects were more intensely colonized than others. In the present investigation, this characteristic was more common in the group of individuals with CP. Additionally, this group of patients had the greatest number of candida albicans diversity index and a greater number of total isolates per species. These differences could be related to the immunological state of the patients. The results of this study was similar to study conducted by Urzu et al in 2014 (CFU determination, Sabouraud dextrose agar plate with tetracycline $(50 \mathrm{mg} / \mathrm{ml})$ and incubated at $37^{0} \mathrm{C}$ for $\left.48 \mathrm{~h}\right){ }^{3}$ In the present study, Candida albicans were isolated. Several yeast species have been isolated from periodontal pockets in patients with periodontitis. These species had been described in other studies (C. albicans, C. tropicalis, C. parapsilosis, C. dubliniensis, C. glabrata, and C.guilliermondii), but to our knowledge this is the first study reporting on the isolation of C.albicans from periodontal pockets using real time PCR (rt-PCR).

\section{Conclusion:-}

The interaction between C. albicans and its host is dynamic and complex as this pathogen exhibits multifaceted strategies for growth, proliferation, and survival within the host accompanied by mechanisms to evade host defense. As stressed by researchers in literature identification of C. albicans should be monitored closely. A better understanding of $\mathrm{C}$. albicans host interactions provides important insights into other fungal pathogens. It expands the scope of developing diagnostic strategies to prevent dissemination through epithelial barriers, reaching the bloodstream and prevent life-threatening. It was concluded that candida albicans were associated with CP and AP as compared to $\mathrm{PH}$ patients favouring further progression of periodontal disease with high occurence of deep pockets.

\section{References:-}

1. Vishakha Grover, Anoop Kapoor, Ranjan Malhotra, Virinder Singh Battu, Archana Bhatia, Sonia Sachdeva. To assess the effectiveness of a chlorhexidine chip in the treatment of chronic periodontitis: A clinical and radiographic study. Journal of Indian Society of Periodontology 2011;15 (2):139-146.

2. Janaina C. O. Sardi, Cristiane Duque, Flavia S. Mariano, Iza T. A. Peixoto, Jose F. Hofling and Reginaldo B. Goncalves.Candida spp. in periodontal disease: a brief review. Journal of Oral Science, 2010;52(2):177-185.

3. B. Urzu A, G. Hermosilla, J. Gamonal, I. Morales-Bozo, M. Canals, S. Barahona,C. CO Ccola \& V. Cifuentes. Yeast diversity in the oral microbiota of subjects with periodontitis: Candida albicans and Candida dubliniensis colonize the periodontal pockets. Medical Mycology 2008; 46:783 - 793.

4. A Ja rvensivu, J Hietanen, R Rautemaa, T Sorsa, M Richardson. Candida yeasts in chronic periodontitis tissues and subgingival microbial biofilms in vivo. Oral Diseases 2004;10:106-112.

5. Supriya H, Harishchandra Rai, Suhasini PD, Rajalekshmi V. Pathogenic Mechanisms of Candida Albicans in Oral Mucosa - A Review. International Journal of Health Sciences \& Research 2016;6(1):489-97.

6. Vanpelt E, Belkum VAV, Hays JP. Principles and Technical aspects of PCR amplification. Springer 2008; 34. 